\title{
NINJ2 SNP may affect the onset age of first-ever ischemic stroke without increasing silent cerebrovascular lesions
}

\author{
Dong-Eog Kim ${ }^{1,24^{*}}$, Sang-Mi Noh ${ }^{1}$, Sang-Wuk Jeong ${ }^{1,2}$ and Min-Ho $\mathrm{Cha}^{3}$
}

\begin{abstract}
Background: To investigate if single nucleotide polymorphisms on chromosome 12p13 and within $11 \mathrm{~kb}$ of the gene NINJ2 would be associated with earlier-onset (vs. late-onset) first-ever ischemic stroke and increase silent cerebrovascular lesions prior to the manifestation of the stroke.

Methods: We prospectively enrolled 164 patients (67.6 \pm 12.9 years, 92 men) admitted with first-ever ischemic strokes. All patients underwent genotyping of rs11833579 and rs12425791 as well as systemic investigations including magnetic resonance (MR) imaging and other vascular workup. Stroke-related MR lesions were registered on a brain-template-set using a custom-built software package 'Image_QNA': high-signal-intensity ischemic lesions on diffusion, T2-weighted, or fluid attenuation inversion recovery (FLAIR) MR images, and low signal intensity hemorrhagic lesions on gradient-echo MR images.

Results: The rs11833579 A/A or G/A genotype was independently associated with the first-ever ischemic stroke before the age 59 vs. 59 or over, after adjusting for cardiovascular risk factors and prior medication of antiplatelet or anticoagulant drugs, increasing the risk by about 2.5 fold. In the quantitative MR lesion maps from age-sex matched subgroups ( $n=124$ or 126 ), there was no difference between the patients with the rs11833579 A/A or G/ A genotype and those with the G/G genotype. Unexpectedly, the extent of leukoaraiosis on FLAIR-MR images tended to be smaller in the corona radiata and centrum semiovale of the patients with the rs $12425791 \mathrm{~A} / \mathrm{A}$ or G/A genotype than in those with the $\mathrm{G} / \mathrm{G}$ genotype $(P=0.052)$. Neither the rs 11833579 nor the $\mathrm{r} 12425791$ genotype significantly affected initial stroke severity; however the latter was associated with relatively low modified Rankin scale scores at 1 year after stroke.

Conclusions: The rs11833579 A/A or G/A genotype may bring forward the onset age of first-ever ischemic stroke without increasing silent cerebrovascular lesions prior to the stroke. Further studies are required to confirm our preliminary findings.
\end{abstract}

\section{Background}

Ischemic Stroke is a leading cause of death and disability worldwide. The South London Stroke Register, a population based study of stroke incidence and outcome, showed that only a third of survivors had returned to paid work at 1 year [1]. According to the results to estimate projected costs of ischemic stroke in the United States, loss of earnings was expected to be the highest contributor to the future economic burden

\footnotetext{
* Correspondence: kdongeog@duih.org

'Department of Neurology, Dongguk University Ilsan Hospital, Dongguk

University College of Medicine, Goyang, Republic of Korea

Full list of author information is available at the end of the article
}

of stroke, constituting nearly one-third of the total costs [2]. This burden is multiplied if the stroke occurs earlier in life. The prediction of the stroke onset age would contribute to better prevention of stroke by allowing more individualized strategies such as control of modifiable vascular risk factors in a more precise and intense manner. However, there is limited information on factors influencing the onset age of first-ever ischemic stroke.

Recently, intergenic single-nucleotide polymorphisms (rs11833579 or rs12425791 SNP) on chromosome 12p13 and within $11 \mathrm{~kb}$ of the gene NINJ2 that encodes ninjurin2, an adhesion molecule expressed in glia and

\section{Biomed Central}


shows increased expression after nerve injury $[3,4]$, were reported to be associated with total, ischemic, and atherothrombotic stroke in white persons [5]. Thereafter, it was followed by controversial results in different populations [6-9]. In the Japanese population, rs12425791 was significantly associated with atherothrombotic stroke: both small artery occlusion and large artery atherosclerosis [6]. However, many studies including a Chinese Han study failed to replicate the association of either rs11833579 or rs12425791with ischemic stroke [7-9]. In the field of stroke, including the NINJ2-related studies, failure to find replicated SNP effects across populationbased studies has significantly limited the utility of genetic association results [10]. Stroke cannot be regarded as a single disease entity but as a heterogeneous syndrome, with more than 150 known causes [11], under multiple genetic and clinical influences; and the effect size of a single SNP could be rather small in predicting stroke risk in the general population. Moreover, roles for a genetic factor in the general population vs. stroke population may not be identical. In addition, the influence of genetic factors might be more pronounced in the younger, before classic cardiovascular risk factors exert their full influence on the disease, although the genetic and other risk factors could work in concert and produce stroke at a later age [12]. Taken together, these led us to reason that clinical implications for the NINJ2 SNP, including a potential effect on the onset age of the first-ever ischemic stroke, need to be further elucidated 'within a carefully characterized stroke population' in a detailed manner that includes quantitative analyses of magnetic resonance (MR) images, by which many of diagnostic and therapeutic decisions are today guided in clinic. Until now, the impact of the NINJ2 SNP on stroke-related lesions on multi-sequence MR images has not been studied.

In the present study, we tried to see if the NINJ2 SNP (A allele) would be associated with earlier-onset (vs. late-onset) first-ever ischemic stroke. This study, conducted in a hospital-based stroke cohort, could be underpowered to detect small effects of the single SNP on the development of the clinical endpoint $[10,13]$. Thus, in an effort to corroborate the associations, we used quantitative data on stroke-related MR lesions [14] as endophenotypes-subclinical phenotypes that are heritable traits, revealing the actions of genes predisposing an individual to develop conditions known to increase the risk of future disease, often having a larger genetic component than clinical disease endpoints, and manifesting years before clinical diagnostic criteria for the disease are met [13]. Considering that unrecognized or covert brain infarcts and white matter lesions are linked to increased risk for stroke [15], we investigated if the NINJ2 A/A or G/A genotype increased silent cerebrovascular lesions such as leukoaraiosis, asymptomatic lacunes, or microbleeds prior to the manifestation of first strokes. Moreover, we studied if the NINJ2 genotypes affected post-stroke functional outcome at 1 year.

\section{Methods}

This study was approved by the institutional review board of Dongguk University Ilsan Hospital (DUIH).

\section{Patients}

Out of 586 consecutive first-ever ischemic stroke patients admitted to our hospital, a community-based academic hospital at a suburban city (Goyang) in Seoul Metropolitan Area, from 2008 February to October 2010 , we prospectively enrolled 164 patients (67.6 \pm 12.9 years, 92 men) who gave written informed consent to "DUIH stroke-genetic-study project". They did not have a history of hemorrhagic stroke, either. All patients underwent systemic investigations including assessment of ischemic cerebral events, medication history, MR imaging with MR angiography, carotid duplex ultrasonography, transthoracic echocardiography, 24 h Holter monitoring, and other routine admission laboratory tests. In selected patients, transesophageal echocardiography or transcranial Doppler bubble study was also performed. Under a standardized protocol, we prospectively collected demographic data, prior medication history, and the presence of vascular risk factors including hypertension, diabetes mellitus, dyslipidemia, heart disease, and smoking.

\section{Risk factor variables and stroke classification}

Hypertension was considered to be present if a subject had one of the following conditions: repeated blood pressure readings above $140 / 90 \mathrm{mmHg}$ at $1 \sim 2$ weeks after stroke onset, a history of hypertension, or use of antihypertensives. Diabetes mellitus was defined as glycated hemoglobin $(\mathrm{HbA} 1 \mathrm{C}) \geq 6.5 \%$, history of diabetes mellitus, or use of diabetic medication. Dyslipidemia was defined as total cholesterol $\geq 240 \mathrm{mg} / \mathrm{dl}$, low density lipoprotein cholesterol $\geq 160 \mathrm{mg} / \mathrm{dl}$, a history of dyslipidemia, or use of lipid lowering agents. Heart disease as potential source of cerebral embolism was considered to be present if a subject had left atrial or ventricular thrombus, atrial fibrillation or sick sinus syndrome, recent myocardial infarction within 1 month, rheumatoid mitral or aortic valve disease, bioprosthetic or mechanical heart valves, chronic myocardial infarction with low ejection fraction less than $28 \%$, symptomatic congestive heart failure with ejection fraction less than $30 \%$, dilated cardiomyopathy, aneurysms or akinetic segments of the left ventricular wall, or nonbacterial thrombotic endocarditis. Smokers included current smokers or those who had stopped smoking for less than 1 
month. Subtypes of index stroke were determined by the consensus of three neurologists as follows; large artery atherosclerosis, small vessel occlusion, cardiac embolism, and ischemic stroke of undetermined etiology, as described in Trial of Org 10172 in Acute Stroke Treatment (TOAST) [16].

\section{Genotyping}

The genomic DNA of each subject was extracted from peripheral blood mononuclear cells using a Type G Genomic DNA Extraction Kit (GeneAll, Seoul, Korea) according to the manufacturer's instructions. Genotyping of rs11833579 or rs12425791 was conducted using ABI BigDye ${ }^{\mathbb{B}}$ Terminator v3.1 Cycle Sequencing Kits (Aplied Biosystems, Carlsbad, California); genotyping primers and representative chromatograms of the NINJ2 SNPs are shown (see Additional file 1: Table S1 and Additional file 2: Figure S1, respectively). Hardy-Weinberg equilibrium tests were conducted to determine whether individual variants were in equilibrium at each locus. Linkage disequilibrium (LD), Lewontin's D' (|D'|), and $r^{2}$ between pairs of bi-allelic loci were measured [17].

\section{Brain MR lesion mapping}

All patients have had their MR lesions prospectively segmented and registered on the ch2better brain template http://www.mccauslandcenter.sc.edu/CRNL/, for which a custom-built software package Image_QNA [14] was used. The software stores the shape and size of each lesion mapped in the standard template coordinate system, available for future analysis. The registered data includes high signal intensity ischemic lesions on diffusion, T2-weighted, or fluid attenuation inversion recovery (FLAIR) MR images, and low signal intensity hemorrhagic lesions on gradient-echo (GE) MR images. While blinded to patient identification, a trained research assistant performed the segmentation and registration under supervision of an experienced neurologist who can correct the data as needed after reviewing every registration output. The Image_QNA-based registration of MR lesions showed low inter- and intra-user variability. Groups of subjects could be visually grouped together by generating lesion accumulation maps, in which summed images are used to display the visual information of many subjects on a single image set $[14,18]$.

\section{Statistical analysis}

We dichotomized the age quartiles a priori into the first quartile (58 and below, earlier-onset group) and the three highest quartiles combined (59 and above, lateonset group), based on the minimum retirement pension age (59) in Korea. Between the groups, we compared the frequency of the NINJ2 SNP (GA or AA alleles) and conventional risk factors including hypertension, diabetes mellitus, dyslipidemia, heart disease, and smoking. After considering the relationship between the age/sex and stroke severity/recovery, we reasoned that age-sex matching was needed in the analysis to see the relationship between the NINJ2 SNP and stroke severity or recovery or quantitative MR data.

The Student's $t$ test was used for comparison of normally-distributed continuous variables between groups. Chi-square test was used to compare proportions between groups. A logistic regression model was used to test statistical significance of the interaction between the NINJ2 SNP and conventional risk factors in predicting the earlier-onset of first-ever ischemic stroke. We also tried to adjust the results for prior medications. In agesex matched samples, multivariate analyses were performed to determine if the NINJ2 SNP was an independent predictor for admission NIHSS score or 1 year $\mathrm{mRS}$ score. All statistical analyses were performed using a software package (SPSS 18.0, Chicago, IL). A value of $P<0.05$ was considered statistically significant.

\section{Results}

Lower stroke onset ages in the patients with the rs11833579 A/A or G/A genotype than in those with the G/G genotype

Out of 586 first-ever ischemic stroke patients admitted to our hospital from 2008 February to October 2010, 164 patients who gave informed consent were enrolled. Between the enrolled patients and non-enrolled patients, there was no significant difference in age $(67.5 \pm 12.4$ years vs. $68.0 \pm 12.2$ years), male gender ( $54.9 \%$ vs. $53.6 \%)$, and initial NIHSS score $(5.0 \pm 4.6$ vs. $5.3 \pm 5.9)$ (all $P>0.05$ ). However, dyslipidemia was more frequent and heart disease was less frequent in the enrolled group than in the non-enrolled group. The use of statin was more frequent and the use of antihypertensive drugs was less frequent in the enrolled group than in the non-enrolled group. Stroke etiologies were statistically different between the groups. Premorbid mRS was higher and 1 year mRS was lower in the enrolled group than in the non-enrolled group (see Additional file 3: Table S2).

Minor allele frequencies (MAFs) of rs11833579 and rs12425791 NINJ2 SNP were 0.19 and 0.06, respectively, which were slightly lower than MAFs of other ethnicities (see Additional file 4: Table S3) registered in the International HapMap http://hapmap.ncbi.nlm.nih.gov. The two SNPs satisfied Hardy-Weinberg equilibrium ( $P$ $>0.05)$. Genotype frequencies of rs 11833579 were $48.2 \%$ (G/G), 43.9\%, (G/A) and 7.9\% (A/A). Those of rs 12425791 were $60.4 \%(\mathrm{G} / \mathrm{G}), 36.0 \%(\mathrm{G} / \mathrm{A})$, and $3.7 \%$ (A/A). The LD coefficients between the two SNPs were 
$\left|\mathrm{D}^{\prime}\right|=0.864$ and $\mathrm{r}^{2}=0.484(P<0.01)$, which suggested that these two SNPs were not linked in the Korean stroke population.

The ages of the patients with the A allele of rs11833579 SNP (G/A or A/A genotype; $65.2 \pm 13.5$ years) were lower than those without (G/G genotype; $69.4 \pm 11.4$ years, $P=0.032)$. The ages of the patients with the A allele of rs12425791 SNP (G/A or A/A genotype; $65.5 \pm 13.3$ year) were lower than those without (G/G genotype; $68.4 \pm 12.2$ year), which however did not reach a statistical significance $(P=0.150)$. Figure 1 shows the difference of cumulative percentage of strokeonset-age between the rs11833579 A/A or G/A genotype group and the G/G genotype group; the gap appears to begin being narrowed around the age of 60 . Around the age of 59, the cumulative frequency of firstever stroke is about two-fold higher in the rs11833579 A/A or GA genotype group than in the G/G genotype group: approximately $40 \%$ vs. $20 \%$. Around the age of 70 , the genetic predisposition to stroke seemed to be overwhelmed by the age effect.

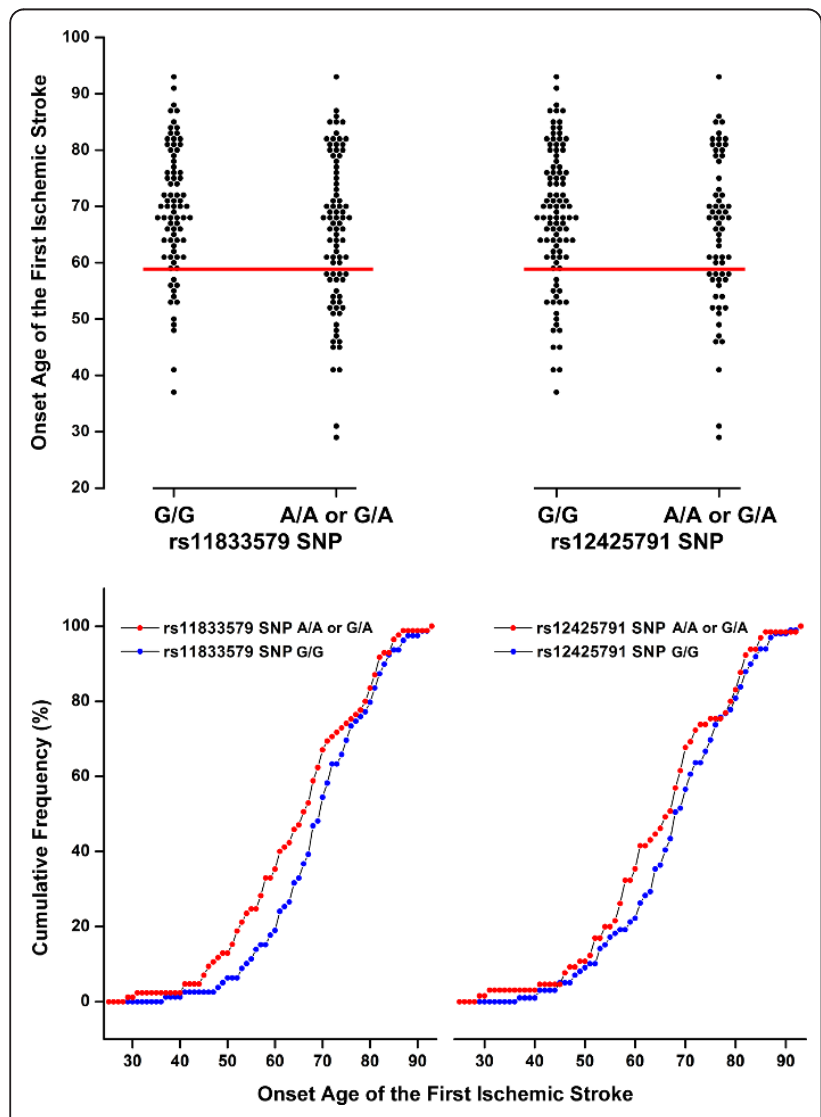

Figure 1 Earlier-onset of first-ever ischemic stroke in the patients with the rs $11833579 \mathrm{G} / \mathrm{A}$ or A/A SNP than in those with the $G / G$ type.
More frequent rs11833579 A/A or G/A genotype in the first-ever ischemic stroke patients aged younger than 59 compared with those aged 59 or older

When the stroke onset age was dichotomized at the first quartile (= age 59), rs11833579 A/A or G/A genotype was more frequently found in the earlier-onset group than in the late-onset group $(28 / 40$ vs. $57 / 124, P=$ 0.008) (Table 1). Rs12425791 A/A or G/A genotype showed a similar tendency $(21 / 40$ vs. $44 / 124, P=0.056)$. In addition, male sex and smoking were more frequent in the earlier-onset group than in the late-onset group. Diabetes mellitus was non-significantly more frequent

Table 1 Univariable analysis: factors associated with earlier-onset (< 59 years) vs.late-onset $(\geq 59)$ first-ever ischemic stroke

\begin{tabular}{|c|c|c|c|}
\hline & $\begin{array}{l}\text { Earlier-onset } \\
\quad(n=40)\end{array}$ & $\begin{array}{l}\text { Late-onset } \\
(n=124)\end{array}$ & $\mathrm{P}^{*}$ \\
\hline$\overline{\text { Age }}$ & $50.2 \pm 7.1$ & $72.8 \pm 8.4$ & $<0.001$ \\
\hline Sex (male) & $34(85.0 \%)$ & $56(45.2 \%)$ & $<0.001$ \\
\hline Rs12425791 genotype (GA or AA) & $21(52.5 \%)$ & $44(35.5 \%)$ & 0.056 \\
\hline Rs11833579 genotype (GA or AA) & $28(70.0 \%)$ & $57(46.0 \%)$ & 0.008 \\
\hline Hypertension & $25(62.5 \%)$ & $91(73.4 \%)$ & 0.188 \\
\hline Diabetes mellitus & $15(37.5 \%)$ & $53(42.7 \%)$ & 0.558 \\
\hline Dyslipidemia & $31(77.5 \%)$ & $79(63.7 \%)$ & 0.107 \\
\hline Heart disease & $1(2.5 \%)$ & $10(8.1 \%)$ & $0.298^{+}$ \\
\hline Smoking & $31(77.5 \%)$ & $55(44.4 \%)$ & $<0.001$ \\
\hline Current smoking & $30(75.0 \%)$ & $50(41.3 \%)$ & $<0.001$ \\
\hline \multicolumn{4}{|l|}{ Prior medication } \\
\hline Antihypertensives & $11(27.5 \%)$ & $52(41.9 \%)$ & 0.103 \\
\hline Antidiabetics & $9(22.5 \%)$ & $38(30.6 \%)$ & $<0.001$ \\
\hline Statins & $10(25.0 \%)$ & $37(29.8 \%)$ & 0.556 \\
\hline Antiplatelet & $8(20.0 \%)$ & $23(18.5 \%)$ & 0.838 \\
\hline Warfarin & $0(0.00 \%)$ & $1(0.81 \%)$ & 0.572 \\
\hline Ischemic stroke subtype ${ }^{\ddagger}$ & & & $0.401^{\dagger}$ \\
\hline Large artery atherosclerosis & $11(27.5 \%)$ & $36(29.0 \%)$ & \\
\hline Small vessel occlusion & $22(55.0 \%)$ & $62(50.0 \%)$ & \\
\hline Cardioembolism & $2(5.0 \%)$ & $4(3.2 \%)$ & \\
\hline Other determined etiology & $1(2.5 \%)$ & $0(0 \%)$ & \\
\hline Undetermined etiology & $4(10.0 \%)$ & $22(17.7 \%)$ & \\
\hline Premorbid mRS & $0.6 \pm 1.2$ & $1.4 \pm 1.8$ & 0.001 \\
\hline Admission NIHSS & $3.1 \pm 2.4$ & $5.5 \pm 5.9$ & $<0.001$ \\
\hline Discharge NIHSS & $1.7 \pm 2.3$ & $3.5 \pm 4.6$ & 0.002 \\
\hline NIHSS (Admission - Discharge) & $1.4 \pm 2.8$ & $2.0 \pm 4.9$ & 0.421 \\
\hline Discharge $\mathrm{mRS}$ & $1.4 \pm 1.3$ & $2.4 \pm 1.7$ & $<0.001$ \\
\hline 1 year mRS $(n=39 \& 117)$ & $0.5 \pm 0.8$ & $2.0 \pm 1.7$ & $<0.001$ \\
\hline
\end{tabular}

Values are number (percentage) or mean \pm standard deviation.

*P for Student's $t$ test or Chi-square test.

${ }^{\dagger}$ Fisher's exact test.

"TOAST classification

mRS and NIHSS denote modified Rankin scale and NIH stroke scale, respectively. 
and prior medication of antidiabetics was significantly more frequent in the late-onset group than in the earlier-onset group. No significant inter-group difference was observed in other vascular risk factors and stroke subtypes. The late-onset group had higher premorbid $\mathrm{mRS}$ scores, more severe strokes, and less favorable outcomes than the earlier-onset group had.

\section{An independent association of rs $11833579 \mathrm{~A} / \mathrm{A}$ or $\mathrm{G} / \mathrm{A}$} genotype and male sex with the first-ever ischemic stroke before the age 59 vs. age 59 or over

To adjust for conventional stroke risk factors a multivariable analysis was performed (Table 2), which showed that rs11833579 A/A or G/A genotype and male sex were independently associated with the first-ever ischemic stroke before the age 59 vs. age 59 or over. In addition, dyslipidemia was associated with the earlieronset stroke, which was marginally significant. When additionally adjusted for prior medication, either antiplatelet or warfarin, in another regression analysis, rs11833579 A/A or G/A genotype and male sex were again positively associated with the earlier-onset of firstever ischemic stroke (see Additional file 5: Table S4).

No significant effect of rs11833579 or rs12425791 SNP on vascular risk factors, stroke etiologies, and initial stroke severity

By matching age and sex while blinded to patient identification, we selected 126 subjects with $(\mathrm{n}=63,68.3 \pm$ 11.7 years, 31 men) or without $(n=63,68.3 \pm 11.0$ years, 32 men) rs11833579 A/A or G/A genotype (see Additional file 6: Table S5). There was no intergroup difference in the vascular risk factors and distribution of ischemic stroke subtypes. Statin medication was more frequent in the rs11833579 A/A or G/A genotype group than in the G/G genotype group with borderline significance $(P=0.052)$. Admission NIHSS scores were non-

Table 2 Multivariable analysis: factors associated with earlier-onset (< 59 years) vs.late-onset $(\geq 59)$ first-ever ischemic stroke

\begin{tabular}{|c|c|c|}
\hline Variable & $\begin{array}{l}\text { Odds ratio ( } 95 \% \text { C.I.) for } \\
\text { the } \\
\text { earlier-onset first stroke }\end{array}$ & $P$ \\
\hline $\begin{array}{l}\text { Rs11833579 genotype (GA or } \\
\text { AA) }\end{array}$ & $2.46(1.06-5.69)$ & 0.036 \\
\hline Male sex & $5.73(1.52-21.61)$ & 0.010 \\
\hline Hypertension & $0.50(0.20-1.23)$ & 0.129 \\
\hline Diabetes mellitus & $0.95(0.40-2.24)$ & 0.904 \\
\hline Dyslipidemia & $2.51(0.98-6.44)$ & 0.057 \\
\hline Heart disease & $0.29(0.03-2.78)$ & 0.283 \\
\hline Smoking & $1.34(0.40-4.49)$ & 0.636 \\
\hline
\end{tabular}

Hosmer-Lemeshow goodness-of-fit test showed $\chi^{2}=11.43$ and $P=0.18$, demonstrating a good fitness of the model. C.I. confidence interval. significantly $(P=0.103)$ lower in the former than in the latter. However, 'admission NIHSS - discharge NIHSS' scores did not show statistical differences between the groups $(P=0.251)$. At 1 year follow up, mRS scores did not differ between the groups, either $(P=0.649)$. Moreover, in a conditional logistic regression analysis for the matched subgroup data, no parameters were significantly associated with the rs11833579 A/A or G/A genotype vs. G/G genotype (see Additional file 7: Table S6). Another multivariable linear regression analysis to adjust for statin medication and premorbid mRS showed that not the rs11833579 A/A or G/A genotype but premorbid mRS was significantly associated with admission NIHSS scores (see Additional file 8: Table S7).

Table 3 Clinical profiles of age-sex matched patients with the rs12425791 A/A or G/A genotype vs.GG genotype

\begin{tabular}{|c|c|c|c|}
\hline & \multicolumn{2}{|c|}{ Rs12425791 genotype } & \multirow[t]{2}{*}{$\mathrm{P}^{*}$} \\
\hline & $\begin{array}{c}(A / A \text { or } G / A) \\
(n=62)\end{array}$ & $\begin{array}{c}(\mathrm{GG}) \\
(\mathrm{n}=62)\end{array}$ & \\
\hline Age & $68.3 \pm 11.7$ & $68.3 \pm 11.0$ & 0.994 \\
\hline Sex (male) & $35(56.5 \%)$ & $34(54.8 \%)$ & 0.857 \\
\hline Hypertension & $42(67.7 \%)$ & $46(74.2 \%)$ & 0.429 \\
\hline Diabetes mellitus & $24(38.7 \%)$ & $24(38.7 \%)$ & 1.000 \\
\hline Dyslipidemia & $41(66.1 \%)$ & $47(75.8 \%)$ & 0.235 \\
\hline Heart disease & $5(8.1 \%)$ & $2(3.2 \%)$ & $0.439^{\dagger}$ \\
\hline Smoking & $32(51.6 \%)$ & $33(53.2 \%)$ & 0.857 \\
\hline Current smoking & 31 (50.0\%) & 29 (46.8\%) & 0.526 \\
\hline
\end{tabular}

Prior medication

\begin{tabular}{lccc}
\hline Antihypertensives & $21(33.9 \%)$ & $23(37.1 \%)$ & 0.707 \\
\hline Antidiabetics & $21(33.9 \%)$ & $24(38.7 \%)$ & 0.575 \\
\hline Statins & $19(30.6 \%)$ & $19(30.6 \%)$ & 1.000 \\
\hline Antiplatelets & $12(19.4 \%)$ & $12(19.4 \%)$ & 1.000 \\
\hline Warfarin & $0(0.0 \%)$ & $1(1.6 \%)$ & $0.496^{\dagger}$ \\
\hline Ischemic stroke subtype & & & $1.000^{\dagger}$
\end{tabular}

\begin{tabular}{lccc}
\hline Large artery atherosclerosis & $16(25.8 \%)$ & $17(27.4 \%)$ & \\
\hline Small vessel occlusion & $35(56.5 \%)$ & $35(56.5 \%)$ & \\
\hline Cardioembolism & $1(1.6 \%)$ & $2(3.2 \%)$ & \\
\hline Other determined etiology & $1(1.6 \%)$ & $0(0.0 \%)$ & \\
\hline Undetermined etiology & $9(14.5 \%)$ & $8(12.9 \%)$ & \\
\hline Premorbid mRS & $1.2 \pm 1.6$ & $1.0 \pm 1.6$ & 0.575 \\
\hline Admission NIHSS & $4.4 \pm 3.6$ & $4.5 \pm 4.1$ & 0.872 \\
\hline Discharge NIHSS & $2.7 \pm 3.7$ & $2.4 \pm 2.9$ & 0.629 \\
\hline NIHSS (admission - discharge) & $1.7 \pm 3.3$ & $2.1 \pm 3.5$ & 0.512 \\
\hline Discharge mRS & $2.1 \pm 1.6$ & $2.0 \pm 1.6$ & 0.612 \\
\hline 1 year mRS $(n=58 \& 62)$ & $1.2 \pm 1.4$ & $1.6 \pm 1.6$ & 0.143
\end{tabular}

Values are number (percentage) or mean \pm standard deviation.

*P for Student's $t$ test or Chi-square test.

${ }^{\dagger}$ Fisher's exact test.

${ }^{\ddagger}$ TOAST classification

mRS and NIHSS denote modified Rankin scale and NIH stroke scale, respectively. 
We also selected 124 age-sex matched subjects with $(\mathrm{n}=$ $62,66.6 \pm 12.0$ years, 35 men) or without $(n=62,66.6 \pm$ 11.1 years, 34 men) the rs12425791 A/A or G/A genotype (Table 3). Between the groups, there was no difference except that 1 year $\mathrm{mRS}$ scores were non-significantly $(P=$ 0.143 ) lower in the rs12425791 A/A or G/A genotype group than in the $\mathrm{G} / \mathrm{G}$ genotype group. In a multivariable analysis after adjusting for premorbid mRS and admission NIHSS that turned out to be a significant predictor $(P<$ 0.001 ) for the mRS score at 1 year, rs12425791 GA or AA genotype was a significant negative predictor of $\mathrm{mRS}$ score at 1 year (Model I of Table 4).

\section{A trend of smaller white matter lesion extent in the} patients with the rs 12425791 A/A or G/A genotype than in those with the G/G genotype

In the quantitative mapping of stroke-related lesions on diffusion/FLAIR/T2/GE MR images, there was no difference between the age-sex matched patients with the rs11833579 A/A or G/A genotype and those with the G/ $\mathrm{G}$ genotype (Figure 2A). Likewise, there was no difference in the MR images of the age-sex matched patients with the rs12425791 A/A or G/A genotype vs. those with the $\mathrm{G} / \mathrm{G}$ genotype, except that the extent of leukoaraiosis in the corona radiata and centrum semiovale tended to be smaller in the former $(1861 \pm 2437$ pixels $)$ than in the latter $(1136 \pm 1528$ pixels, $P=0.052)$ (Figure $2 \mathrm{~B}$ ).

When the extent of leukoaraiosis and onset age of stroke, two parameters that could have potential links with the NINJ2 SNP or post-stroke prognosis, were additionally entered into the multivariable analysis model to predict mRS at 1 year (Model I of Table 4), the SNP lost significance whereas the age was a statistically significant predictor and the leukoaraiosis burden was a marginally significant predictor for the follow-up mRS (Model II of table 4).

\section{Discussion and Conclusions}

In the present study, we found that the rs11833579 A/A or G/A NINJ2 genotype was an independent risk factor for the first-ever ischemic stroke before the age 59 vs. age 59 or over, increasing the relative risk by about 2.5 fold. First strokes occurred before the age 59 in $70 \%$ of the patients with the A/A or G/A genotype, but only in about $46 \%$ of the patients with the G/G genotype. Male sex was another risk factor for the earlier-onset of first stroke, which is in line with a previous report [19-22]. In the quantitative mapping of stroke-related lesions on multi-sequence MR images from age-sex matched subjects, there was no difference between the patients with the rs11833579 A/A or G/A genotype and those with the G/G genotype. Both the rs11833579 and rs12425791 genotypes did not significantly affect initial stroke severity or subsequent recovery from the stroke.

Meschia et al. reported that family history of stroke before the age of 65 years was a stronger risk factor at age 65 years or earlier, which indicates that an inherited component of stroke risk may attenuate with age [23-25]. Thus, once risk factor genes are identified, it would be informative to assess relative risk for stroke as a function of age $[26,27]$. We found that around the age sixty the genetic predisposition to stroke by the rs11833579 A/A or G/A genotype started being attenuated by the age effect. After adjusting for conventional risk factors and prior medication of antiplatelet or anticoagulant drugs, the association between the rs11833579 A/A or G/A genotype and the earlier-onset of first ischemic stroke remained significant. Between the agesex matched subgroups with vs. without the rs11833579 A/A or G/A genotype, there was no difference in the extent and distribution of silent cerebrovascular lesions as well as of acute ischemic infarction. In addition, there was no intergroup difference in the vascular risk factors

Table 4 Multivariable analyses to predict modified Rankin scale (mRS) score at $\mathbf{1}$ year

\begin{tabular}{ccc}
\hline Variable & Odds ratio (95\% C.I.) for mRS score & P \\
\hline Model I & & \\
\hline Rs12425791 (GA or AA) & $-0.15(-1.01-0.04)$ & 0.036 \\
\hline Premorbid mRS & $0.09(-0.07-0.24)$ & 0.262 \\
\hline Admission NIHSS & $0.38(0.08-0.20)$ & $<.001$ \\
\hline Model II & & 0.230 \\
\hline Rs12425791 (GA or AA) & $-0.080(-0.618-0.150)$ & 0.825 \\
\hline Premorbid mRS & $0.016(-0.122-0.152)$ & $<0.001$ \\
\hline Admission NIHSS & $0.323(0.068-0.171)$ & $<0.001$ \\
\hline Age & $0.369(0.030-0.067)$ & 0.068 \\
\hline Pre-stroke leukoraiosis burden (per 100 pixels) & $0.128(0.000-0.016)$ & \\
\hline
\end{tabular}

NIHSS and C.I. denote NIH stroke scale and confidence interval, respectively.

Leukoaraiosis burden: extent of leukoaraiosis in the corona radiata and centrum semiovale on fluid attenuation inversion recovery (FLAIR) magnetic resonance images 


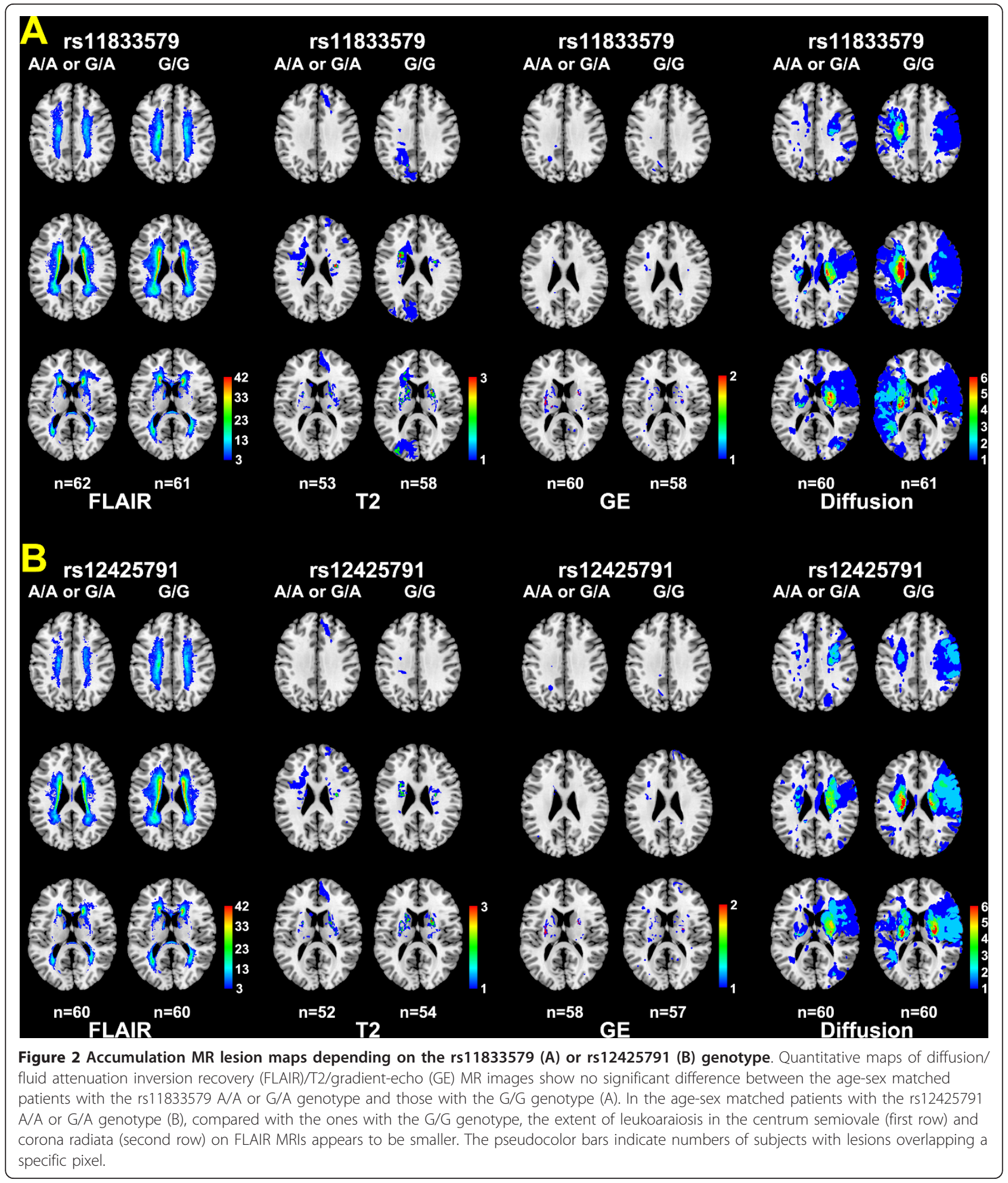

and distribution of ischemic stroke subtypes. These may explain the results that initial stroke severity or subsequent recovery from the stroke did not differ between the groups.
The Framingham Heart Study showed that the presence of severe leukoaraiosis at baseline more than doubles the future risk of stroke [28]. Many studies showed that leukoaraiosis volume, influenced by genetic 
variation in several underlying biological processes, was highly heritable [29-31]. In our study, the burden of leukoaraiosis did not differ between the patients with the rs11833579 A/A or G/A genotype and those with the G/G genotype. The mean onset age of first-ever ischemic stroke was significantly but modestly (about 4 years) younger in the patients with the A allele than in those without. These suggest that the genetic factor might work in concert with other classic risk factors $[12,32]$ that do not affect leukoaraiosis, or induce stroke before the conventional vascular risk factors exert their full influence on the leukoaraiosis. It should be understood in a similar vein that NINJ2 SNPs were not associated with an increased risk of the other covet cerebrovascular lesions-clinically silent infarcts or hemorrhage-either.

We found a trend of earlier-onset stroke in the patients with the rs12425791 A/A or G/A genotype compared with those with the G/G genotype; however, the extent of leukoaraiosis in the corona radiata and centrum semiovale tended to be smaller in the age-sex matched patients with the A/A or G/A genotype than in those with the G/G genotype. The weak association of the SNP with lower leukoaraiois burden as well as with earlier-onset stroke should be further investigated in future research. The association might be a mere coincidence, however it again suggests that aggravation of small vessel disease may not be the cause of the SNPassociated earlier-onset of first-ever ischemic stroke. The Greater Cincinnati Stroke Study [33] and a single center study from Massachusetts General Hospital [34] showed that, when compared with no or mild leukoaraiosis, severe leukoaraiosis was a strong predictor of a higher mRS score at 3 or 6 months. Thus, the rs12425791 A/A or G/A genotype-related attenuation of leukoaraisosis, along with the non-significantly earlier onset of the first stroke, might have contributed to relatively low post-stroke mRS scores at 1 year. This may be supported by the results of the two-step multivariable analysis (Table 4), which showed that the NINJ2 SNP lost significance after entering the 'extent of leukoaraiosis' and 'onset age of stroke' into the model.

There have been conflicting reports on the association between the NINJ2 SNP and ischemic stroke [5-8]. This may be due to heterogeneity among different ethnic groups or false positive or false negative results, possibly being related to the small effect size of the SNP in terms of predicting the stroke risk in the general population. The present study conducted within Korean stroke patients, while having characterized the subjects in a detailed manner with prospective registration of not only alphanumeric data but also a comprehensive set of quantitative multi-sequence MR imaging data [14], may have selection bias; and, our findings need to be replicated in other population samples. In addition, our short-term cohort study did not investigate the duration of exposure to cardiovascular risk factors, quality of control of the risk factors, and environmental factors that could influence the onset age of first stroke. Despite the limitations, as a hypothesis-generating research, our study to provide with both genetic and quantitative-MR data as well as post-stroke functional outcome data merits further confirmatory investigation with a larger sample size.

To summarize, the rs11833579 A/A or G/A NINJ2 genotype may bring forward the onset age of first-ever ischemic stroke without affecting asymptomatic cerebrovascular lesion formation prior to the stroke and neurological and radiological severity of the stroke, and functional recovery after the stroke. The rs12425791 A/ A or G/A genotype was associated with relatively low post-stroke mRS scores at 1 year.

\section{Additional material}

\begin{abstract}
Additional file 1: Table S1. Genotyping primers of rs12425791 and rs11833579 NINJ2 single nucleotide polymorphisms (SNPs).

Additional file 2: Figure S1. Representative chromatograms of NINJ2 single nucleotide polymorphisms genotyping.

Additional file 3: Table S2. Clinical profiles of non-enrolled vs. enrolled subjects.

Additional file 4: Table S3. Characteristics of NINJ2 SNPs with MAFs of the study population (Korean stroke subjects) vs. other ethnic populations.

Additional file 5: Table S4. Multivariable analysis: factors associated with earlier-onset ( $<59$ years) vs. late-onset $(\geq 59)$ first-ever ischemic stroke.

Additional file 6: Table S5. Clinical profiles of age-sex matched patients with the rs11833579 A/A or G/A genotype vs. G/G genotype.

Additional file 7: Table S6. Conditional logistic regression analysis to find independent factors associated with the rs11833579 A/A or G/A genotype vs. G/G genotype.

Additional file 8: Table S7. Multivariable analysis to predict admission $\mathrm{NIH}$ Stroke Scale (NIHSS) score.
\end{abstract}

\section{Acknowledgements}

This study was supported by a grant (A08020) of the Korea Healthcare Technology R\&D Project, Ministry of Health \& Welfare, Republic of Korea. We thank Dr. II-Young Jung and Stroke Research Nurses (Eun-Kyoung Hwang, Dong-Hee Kang, and Jeong-Eun Park) for prospective data collection.

\section{Author details}

${ }^{1}$ Department of Neurology, Dongguk University Ilsan Hospital, Dongguk University College of Medicine, Goyang, Republic of Korea. ${ }^{2}$ Molecular Imaging and Neurovascular Research (MINER) Laboratory, Dongguk University Ilsan Hospital, Goyang, Republic of Korea. ${ }^{3}$ Brain Research Center, Korea Institute of Oriental Medicine, Daejeon, Republic of Korea. ${ }^{4}$ Division of Stroke Medicine, Department of Neurology, Dongguk University IIsan Hospital, 814 Siksa-dong, Goyang 410-773, Republic of Korea.

\section{Authors' contributions}

DEK conceived and designed the study. He recruited the patients and interpreted the study data, and wrote a draft. SMN and SWJ recruited the patients and participated in the intellectual discussion of the results and 
critical revision of the manuscript. They also participated in study design and coordination. MHC interpreted the data and participated in the intellectual discussion of the results and critical revision of the manuscript. All authors read and approved the final manuscript. Each author has participated sufficiently in the work to take public responsibility for appropriate portions of the content. All authors read and approved the final manuscript.

\section{Competing interests}

The authors declare that they have no competing interests.

Received: 1 December 2011 Accepted: 20 March 2012

Published: 20 March 2012

\section{References}

1. Busch MA, Coshall C, Heuschmann PU, McKevitt C, Wolfe CD: Sociodemographic differences in return to work after stroke: the South London Stroke Register (SLSR). J Neurol Neurosurg Psychiatry 2009, 80:888-893.

2. Brown DL, Boden-Albala B, Langa KM, Lisabeth LD, Fair M, Smith MA, Sacco RL, Morgenstern LB: Projected costs of ischemic stroke in the United States. Neurology 2006, 67:1390-1395.

3. Araki T, Milbrandt J: Ninjurin2, a novel homophilic adhesion molecule, is expressed in mature sensory and enteric neurons and promotes neurite outgrowth. J Neurosci 2000, 20:187-195.

4. Zhang S, Dailey GM, Kwan E, Glasheen BM, Sroga GE, Page-McCaw A: An MMP liberates the Ninjurin A ectodomain to signal a loss of cell adhesion. Genes Dev 2006, 20:1899-1910.

5. Ikram MA, Seshadri S, Bis JC, Fornage M, DeStefano AL, Aulchenko YS, Debette S, Lumley T, Folsom AR, van den Herik EG, et al: Genomewide association studies of stroke. N Engl J Med 2009, 360:1718-1728.

6. Matsushita T, Umeno J, Hirakawa Y, Yonemoto K, Ashikawa K, Amitani H, Ninomiya T, Hata J, Doi Y, Kitazono T, et al: Association study of the polymorphisms on chromosome 12p13 with atherothrombotic stroke in the Japanese population. J Hum Genet 2010, 55:473-476.

7. Ding H, Tu X, Xu Y, Xu C, Wang X, Cui G, Bao X, Hui R, Wang QK, Wang DW: No evidence for association of 12p13 SNPs rs11833579 and rs12425791 within NINJ2 gene with ischemic stroke in Chinese Han population. Atherosclerosis 2011, 216:381-382.

8. Failure to validate association between $12 \mathrm{p} 13$ variants and ischemic stroke. N Engl J Med 2010, 362:1547-1550.

9. Lotta LA, Giusti B, Saracini C, Vestrini A, Volpe M, Rubattu S, Peyvandi F: No association between chromosome $12 \mathrm{p} 13$ single nucleotide polymorphisms and early-onset ischemic stroke. J Thromb Haemost 2010, 8:1858-1860.

10. Smith JA, Turner ST, Sun $\mathrm{V}$, Fornage M, Kelly RJ, Mosley TH, Jack CR, Kullo IJ, Kardia SL: Complexity in the genetic architecture of leukoaraiosis in hypertensive sibships from the GENOA Study. BMC Med Genomics 2009, 2:16.

11. Amarenco P, Bogousslavsky J, Caplan LR, Donnan GA, Hennerici MG Classification of stroke subtypes. Cerebrovasc Dis 2009, 27:493-501.

12. Brenner DA, Alberts MJ, Amarenco P: Clinical genetic issues in stroke. In Handbook of Clinical Neurology Stroke Part I: Basic and Epidemiological Aspects.. 3 edition. Edited by: Fisher M. Amsterdam: Elsevier B.V; 2009:355-372.

13. Seshadri S, DeStefano AL, Au R, Massaro JM, Beiser AS, Kelly-Hayes M Kase CS, D'Agostino RB Sr, Atwood LD, Decarli C: Genetic correlates of brain aging on MRI and cognitive test measures: a genome-wide association and linkage analysis in the Framingham Study. BMC Med Genet 2007, 8:S15.

14. Kim DE, Park KJ, Schellingerhout D, Jeong SW, Ji MG, Choi WJ, Tak YO, Kwan GH, Koh EA, Noh SM, et al: A new image-based stroke registry containing quantitative MRI data. Cerebrovasc Dis 2011, 32:567-576.

15. Vermeer SE, Hollander M, van Dijk EJ, Hofman A, Koudstaal PJ, Breteler MM: Silent brain infarcts and white matter lesions increase stroke risk in the general population: the Rotterdam Scan Study. Stroke 2003, 34:1126-1129.

16. Adams HP: Design of the trial of org 10172 in acute stroke treatment (TOAST). Control Clin Trials 1997, 18:358-377.

17. Hedrick PW: Gametic disequilibrium measures: proceed with caution. Genetics 1987, 117:331-341.

18. Kim DE, Kim JY, Schellingerhout D, Kim EJ, Kim HK, Lee S, Kim K, Kwon IC, Shon SM, Jeong SW, et al: Protease imaging of human atheromata captures molecular information of atherosclerosis, complementing anatomic imaging. Arterioscler Thromb Vasc Biol 2010, 30:449-456.

19. Vannucci SJ, Hurn PD: Gender differences in pediatric stroke: is elevated testosterone a risk factor for boys? Ann Neurol 2009, 66:713-714.

20. Mercuro G, Deidda M, Piras A, Dessalvi CC, Maffei S, Rosano GM: Gender determinants of cardiovascular risk factors and diseases. J Cardiovasc Med (Hagerstown) 2010, 11:207-220

21. Paolucci S, Bragoni M, Coiro P, De Angelis D, Fusco FR, Morelli D, Venturiero $V$, Pratesi $L$ : Is sex a prognostic factor in stroke rehabilitation? A matched comparison. Stroke 2006, 37:2989-2994.

22. Petrea RE, Beiser AS, Seshadri S, Kelly-Hayes M, Kase CS, Wolf PA: Gender differences in stroke incidence and poststroke disability in the Framingham heart study. Stroke 2009, 40:1032-1037.

23. Meschia JF, Case LD, Worrall BB, Brown RD Jr, Brott TG, Frankel M, Silliman S, Rich SS: Family history of stroke and severity of neurologic deficit after stroke. Neurology 2006, 67:1396-1402.

24. Meschia JF: Clinically translated ischemic stroke genomics. Stroke 2004, 35:2735-2739.

25. Meschia JF, Atkinson EJ, O'Brien PC, Brott TG, Brown RD Jr, Hardy J: Familial clustering of stroke according to proband age at onset of presenting ischemic stroke. Stroke 2003, 34:e89-91.

26. Meschia JF: Ischemic stroke as a complex genetic disorder. Semin Neurol 2006, 26:49-56

27. Lansbury AJ, Grant PJ, Catto AJ: Atherothrombotic risk factors in subjects with a family history of stroke. Cerebrovasc Dis 2002, 14:153-160.

28. Debette S, Beiser A, DeCarli C, Au R, Himali JJ, Kelly-Hayes M, Romero JR, Kase CS, Wolf PA, Seshadri S: Association of MRI markers of vascular brain injury with incident stroke, mild cognitive impairment, dementia, and mortality: the Framingham Offspring Study. Stroke 2010, 41:600-606.

29. Turner ST, Jack CR, Fornage M, Mosley TH, Boerwinkle E, de Andrade M: Heritability of leukoaraiosis in hypertensive sibships. Hypertension 2004, 43:483-487.

30. Carmelli D, DeCarli C, Swan GE, Jack LM, Reed T, Wolf PA, Miller BL: Evidence for genetic variance in white matter hyperintensity volume in normal elderly male twins. Stroke 1998, 29:1177-1181.

31. Atwood LD, Wolf PA, Heard-Costa NL, Massaro JM, Beiser A, D'Agostino RB, DeCarli C: Genetic variation in white matter hyperintensity volume in the Framingham Study. Stroke 2004, 35:1609-1613.

32. $\mathrm{XuX}$, Li J, Sheng W, Liu L: Meta-analysis of genetic studies from journals published in China of ischemic stroke in the Han Chinese population. Cerebrovasc Dis 2008, 26:48-62.

33. Kissela B, Lindsell CJ, Kleindorfer D, Alwell K, Moomaw CJ, Woo D, Flaherty ML, Air E, Broderick J, Tsevat J: Clinical prediction of functional outcome after ischemic stroke: the surprising importance of periventricular white matter disease and race. Stroke 2009, 40:530-536.

34. Arsava EM, Rahman R, Rosand J, Lu J, Smith EE, Rost NS, Singhal AB, Lev MH, Furie KL, Koroshetz WJ, et al: Severity of leukoaraiosis correlates with clinical outcome after ischemic stroke. Neurology 2009, 72:1403-1410.

doi:10.1186/1756-0500-5-155

Cite this article as: Kim et al: NINJ2 SNP may affect the onset age of first-ever ischemic stroke without increasing silent cerebrovascular lesions. BMC Research Notes 2012 5:155.

\section{Submit your next manuscript to BioMed Central and take full advantage of:}

- Convenient online submission

- Thorough peer review

- No space constraints or color figure charges

- Immediate publication on acceptance

- Inclusion in PubMed, CAS, Scopus and Google Scholar

- Research which is freely available for redistribution

Submit your manuscript at www.biomedcentral.com/submit
C Biomed Central 